\title{
Temporal Changes in Cyclin D-CDK4/CDK6 and Cyclin E-CDK2 Pathways: Implications for the Mechanism of Deficient Decidualization in an Immune-Based Mouse Model of Unexplained Recurrent Spontaneous Abortion
}

\section{Zhuo Chang ( $\nabla 1650843841 @ q q . c o m)$}

Heilongjiang University of Traditional Chinese Medicine: Heilongjiang University of Chinese Medicine https://orcid.org/0000-0002-1817-4530

\section{Hai-xue Kuang}

Heilongjiang University of Chinese Medicine

\section{Xueming Zhou}

Heilongjiang University of Chinese Medicine

Hui Zhu

Heilongjiang University of Chinese Medicine

\section{Yang Zhang}

Heilongjiang University of Chinese Medicine

\section{Yin Fu}

Heilongjiang University of Chinese Medicine

\section{Qiang Fu}

Heilongjiang University of Chinese Medicine

Bei Jiang

Heilongjiang University of Chinese Medicine

\section{Wei Wang}

First affiliated hospital of heilongjiang university of chinese medicine

\section{Sha Jiang}

zhuhai hospital of integrated traditional Chinese and Western Medicine

\section{Li Ren}

Hospital of Traditional Chinese Medicine OF Qiqihar

\section{Lei Ma}

Zhaoqing Hospital of Traditional Chinese Medicine

\section{Xue Pan}

Guang'anmen Hospital,Chinese Academy of Chinese Medicine Science

Xiao-ling Feng 


\section{Research Article}

Keywords: Unexplained recurrent spontaneous abortion, Decidualization, Abortion, Cytokines, Cell cycle.

Posted Date: January 12th, 2022

DOI: https://doi.org/10.21203/rs.3.rs-1014689/v2

License: (c) (i) This work is licensed under a Creative Commons Attribution 4.0 International License. Read Full License 


\section{Abstract}

Deficient endometrial decidualization has been associated with unexplained recurrent spontaneous abortion (URSA). However, the underlying mechanism is poorly understood. Here, we aimed to investigate the temporal cytokine changes and the involvement of the cyclin D-cyclin-dependent kinase (CDK)4/CDK6 and cyclin E-CDK2 pathways in the regulation of the G1 phase of the cell cycle during decidualization in a murine model of URSA. Serum and decidual tissues of URSA group and normal pregnant (NP) group mice were collected from gestation day 4 (GD4) to GD8. The embryo resorption and abortion rates were observed on GD8 and the decidual tissue status was assessed using hematoxylin and eosin staining. Cytokine levels in decidual tissues were analyzed using western blotting and reverse transcription polymerase chain reaction. We found that the embryo resorption rate was significantly increased in the URSA group compared to that in the NP group on GD8. The expression of the decidualization marker prolactin in the serum and decidual lysate of the URSA group was significantly decreased on GD6-8 compared to that of the NP group. Cyclin D, CDK4, CDK6, cyclin E, CDK2 and pRb levels in the URSA group mice were significantly lower compared to those in the NP group mice on GD6-8. Our results suggest that the hyperactivated cyclin D-CDK4/CDK6 and cyclin E CDK2 pathways inhibit the decidualization process on GD4, leading to deficient decidualization on GD8. Moreover, they clarify the role of cytokines in the cyclin D-CDK4/6 and cyclin E-CDK2 pathways during decidualization and provide new insight into URSA pathogenesis.

\section{Introduction}

Recurrent spontaneous abortion (RSA) is defined as two or more consecutive pregnancy losses before 20 weeks of gestation [1]. The etiology of RSA is multifactorial, and the influencing factors include anatomical malformations and infections, endocrine dysfunction, prothrombotic, and parental chromosomal disorders $[2,3,4]$. Unexplained recurrent spontaneous abortion (URSA) accounts for approximately $15 \%$ [5] of RSA cases and is characterized as pathological pregnancy with unexplained pathogenesis excluding known factors. Thus, there is an unmet need to explore the mechanism underlying URSA, an unsolved challenge in reproductive medicine. It has been reported that URSA may be related to deficient decidualization ascribed to abnormal changes during the decidualization process [6]. Decidualization is a crucial link required for the successful establishment and maintenance of pregnancy. Decidualization is defined as the cyclic changes the endometrium undergoes in response to stimulation by multiple hormones [7]. During this process, endometrial stromal cells (ESCs) proliferate and transform from spindle-shaped fibroblastic cells into large, round, and multinucleated decidual stromal cells (DSCs) [8]. DSCs acquire a secretory epithelioid-like phenotype after transformation and secrete prolactin (PRL), a functional marker reflecting the level of decidualization [9]. In humans, decidualization is initiated in the mid-secretory phase of each menstrual cycle (day 23) and the decidua is maintained if pregnancy occurs. Briefly, in the absence of implanted embryo in the uterus, the human decidua is routinely formed and then shed off. Alternatively, in rodents, this process is initiated after embryo implantation $[10,11]$. The cellular changes that result from decidualization promote and produce various growth factors, hormones and 
cytokines needed to assure an ongoing pregnancy $[12,13]$. Therebefore, the exploration of abnormal changes during decidualization in URSA samples may offer new insights into the pathogenesis of this disease and affect the relevant therapeutic approaches.

It has been shown that pregnancy loss is closely related to deficient decidualization and that this association is ascribed to abnormal changes during decidualization $[14,15,16,17]$. Decidualization is crucial for the successful establishment and maintenance of pregnancy. Decidualization is defined as the endometrium undergoes cyclic changes in response to multiple hormones. During the process, ESCs proliferate and transform from spindle-shaped fibroblastic cells into large, round, and multinucleated DSCs $[18,19]$. DSCs acquire a secretory epithelioid-like phenotype after transformation and PRL is a crucial maker reflecting the level of decidualization [20]. In humans, decidualization is initiated in the midsecretory phase of each menstrual cycle (day 23 of menstrual cycle) and will maintain the decidua if pregnancy. Differently, this process is initiated after embryo implantation in mice [21]. The cellular change as results of decidualization product various growth factors, hormones, cytokines needed to assure an ongoing pregnancy.

Decidualization involves a large number of cell cycle events, such as cell proliferation, aging and apoptosis, and this process is believed to be regulated through complex signaling mechanisms orchestrated by cell cycle genes [22,23]. The cell cycle is tightly regulated at two specific checkpoints, namely, the G1-S and G2-M phases. The normal operation of these phases is regulated by a complex interplay of cyclins and cyclin-dependent kinases (CDKs) [24]. During cell cycle transitions, different cyclins mediate their actions as positive growth regulators by associating with specific CDKs [25]. Cyclin $D$ and cyclin E associate with their specific CDKs, including CDK4, CDK6 and CDK6, and this is particularly important for the transition from the $\mathrm{G} 1$ to the $S$ phase. The cyclin-CDK complexes orchestrate the cell cycle process by phosphorylating retinoblastoma protein $(\mathrm{Rb})$ as their downstream factor in the cyclin D-CDK4/CDK6 or cyclin E-CDK2 signaling pathway. Some studies have shown that overexpression of cyclin $D$ at the site of implantation improves decidualization defects by shortening the $\mathrm{G} 1$ phase and allowing rapid entry into the $\mathrm{S}$ phase [26]. Furthermore, cyclin $\mathrm{E}$ has been proved to play an active regulatory role in decidualization [27]. However, there is still an unmet need to explore the abnormal expression of these cytokines in URSA and the relationship between these changes and the disease.

This study aimed to investigate the temporal changes of cytokines in cyclin D-CDK4/6 and cyclin E-CDK2 pathways during decidualization, and to analyze the effect of these cytokines on this process.

Furthermore, we explored the connection between abnormal cytokine changes and the high abortion rate of URSA. The purpose of this study was to further characterize the molecular mechanism underlying URSA.

\section{Materials And Methods}

1.Animals 
Ten male DBA/2, 10 male BALB/c and 50 female CBA/J mice (age, 6-8-week-old; weight, 16-22 g) were used in this study. CBA/J mice were purchased from Beijing Huafukang Biotechnology Co., Ltd., and BALB/c and DBA/2 mice were purchased from Beijing Charles River Laboratory Animal Technology Co., Ltd. All animals were housed in our center's animal facility (Centro de experimental animal, Heilongjiang University of traditional Chinese Medicine) under stable humidity $(47 \%)$ and temperature $\left(22-24^{\circ} \mathrm{C}\right)$ conditions on a 14:10-h light/dark cycle, with free access to drinking water and food. All steps were in full compliance with current regulations on the maintenance and use of experimental animals.

\subsection{Pregnancy model and groups}

Virgin female CBA/J mice were mated with male BALB/c mice (normal pregnant [NP] group) or DBA/2 male mice (URSA group). All CBA/J mice were examined at 10:00 am every day. The occurrence of two phenomena was judged as pregnancy success: 1) the vaginal plug was observed, 2) sperm were observed in the vaginal smear of the female CBA/J mice without vaginal plug. The day the sperm or vaginal plug was observed was considered gestation day 1 (GD1).

\subsection{Sample collection}

Pregnant female mice were sacrificed on days 4, 5, 6, 7 and 8 of pregnancy. Mice were sacrificed using pentobarbital sodium anesthesia followed by cervical dislocation. At necropsy, uteri were excised, trimmed of fat, washed with saline, and uterine tissues were collected. To confirm that there were no blastocysts in the collected uterine, two uterine horns were flushed with saline repeatedly. After confirming that there was no embryo residue, the decidual tissue was collected. Half of these decidual tissues were stored at $-80^{\circ} \mathrm{C}$ and the rest were fixed with $4 \%$ paraformaldehyde for total RNA or protein extraction and fixed for preparing the paraffin section.

\section{Hematoxylin and eosin (H\&E) staining}

The reserved decidual tissues were removed from $4 \%$ paraformaldehyde and sectioned, followed by paraffin embedding ( $2 \mathrm{~min}$ ). The paraffin-embedded sections were deparaffinized with xylene (10 min, twice), and rehydrated using descending ethanol concentrations (100\%, $5 \mathrm{~min} ; 95 \%, 2 \mathrm{~min}, 80 \%, 2 \mathrm{~min}$; $70 \%, 2 \mathrm{~min}$ ) and running water ( $2 \mathrm{~min}$ ). After hematoxylin staining for $10 \mathrm{~min}$, the sections were washed with running water. Differentiation was performed in differentiation medium for $30 \mathrm{~s}$. The stained tissue sections were immersed in warm water $\left(50^{\circ} \mathrm{C}\right)$ for $10 \mathrm{~min}$, stained with eosin for $1 \mathrm{~min}$, and washed with running water. After staining with $\mathrm{H} \& \mathrm{E}$, the sections were dehydrated using an increasing alcohol gradient and cleared with xylene. Finally, the slides mounted with neutral balsam were observed under an Olympus BX60 microscope and photographed with a 3D HISTECH Pannoramic250 Panorama Scanner. Three sections per uterine sample were randomly selected for observation. The mean endometrial thickness of the three sections was then considered as the final endometrial thickness of a representative sample from a particular group.

\section{Elisa}


After PBS was added in proportion to decidual tissue, the tissue was dissociated with a homogenizer and homogenized ( $500 \mu \mathrm{l}$ PBS was added for every $10 \mathrm{mg}$ decidual tissue). After centrifugation ( $3000 \mathrm{r} / \mathrm{min}$, $20 \mathrm{~min}, 4^{\circ} \mathrm{C}$ ), the supernatant was taken for standby. Standardized operation was carried out according to the operation methods in the manual.

\section{Immunohistochemistry}

Decidual samples were immediately placed into a 10\% formaldehyde solution bag and fixed for 10 to 24 h. Paraffin blocks were sectioned into a $5 \mu \mathrm{m}$ thickness. The EnVision two-step immunohistochemical staining technique was used to detect the expression of PRL in the decidua. The immunohistochemical staining was performed by one person only. Positive controls were included in every batch of tests. Rabbit anti-rat PRL (Catalog: ab188229, Abcam, Co., Ltd) was used as the primary antibody. An anti-rabbit HRP/DAP Detection kit (Abcam, Co., Ltd) was used for primary antibody detection. Mouse and rat normal decidual tissues were used as PRL positive controls.

\section{Reverse transcription PCR (RT-PCR)}

Total RNA was extracted from decidual tissues using the TRIzol Reagent procedure (Thermo Fisher, 15596026), in accordance with the manufacturer's instructions. Reverse transcription was performed after the total RNA concentration was determined using $2 \mu \mathrm{g}$ of total RNA. cDNA was generated using the reverse transcription kit, according to the manufacturer's instructions. Briefly, RNA was incubated with reverse transcriptase for $15 \mathrm{~min}$ at $37^{\circ} \mathrm{C}$, followed by inactivation of the enzyme at $85^{\circ} \mathrm{C}$ for $5 \mathrm{~s}$. To detect the target cytokine transcripts, PCR was performed using Takara's 2x SYBR Green qPCR Mix Kit (Takara, RR820A). The $20 \mu \mathrm{l}$ reaction system consisted of $1 \mu \mathrm{l}$ of cDNA, $10 \mu \mathrm{l}$ of SYBR®Premix ex Taq II (TLI RNaseH plus), $1.6 \mu \mathrm{l}$ of primers (Table 1 ) and $7.4 \mu \mathrm{l}$ of ultrapure water. Cycling conditions consisted of 45 cycles with denaturation steps at $95^{\circ} \mathrm{C}$ for $30 \mathrm{~s}$, hybridization steps at $95^{\circ} \mathrm{C}$ for $5 \mathrm{~s}$, and an extension step at $60^{\circ} \mathrm{C}$ for $30 \mathrm{~s}$. The $\beta$-actin and c-Met RT-PCR reactions were performed simultaneously, under the same conditions. 
Table 1

Upstream and downstream primers for target gene detection using PCR

\begin{tabular}{|c|c|c|}
\hline Gene Name & Gene Primer & Sequence $\left(5^{\prime}\right.$ to $\left.3^{\prime}\right)$ \\
\hline \multirow[t]{2}{*}{ CDK2 } & Forward Primer & TCCGGATCTTTCGGACTCTG \\
\hline & Reverse Primer & ACAAGCTCCGTCCATCTTCA \\
\hline \multirow[t]{2}{*}{ CDK6 } & Forward Primer & TTGTGACAGACATCGACGAG \\
\hline & Reverse Primer & GACAGGTGAGAATGCAGGTT \\
\hline \multirow[t]{2}{*}{ CDK4 } & Forward Primer & CCAGGCAGGCTTTTCATTCA \\
\hline & Reverse Primer & AGGTCCTGGAAGTATGGGTG \\
\hline \multirow[t]{2}{*}{ Cyclin D } & Forward Primer & GGGGACAACTCTTAAGTCTCAC \\
\hline & Reverse Primer & CCAATAAAAGACCAATCTCTC \\
\hline \multirow[t]{2}{*}{ Cyclin E } & Forward Primer & GAGCTTGAATACCCTAGGACTG \\
\hline & Reverse Primer & CGTCTCTCTGTGGAGCTTATAGAC \\
\hline \multirow[t]{2}{*}{ RB1 } & Forward Primer & ATTCCCTAGTTCACCCTTACGG \\
\hline & Reverse Primer & GATCCTCATTTСTCTTCCTTGTT \\
\hline \multirow[t]{2}{*}{ GAPDH } & Forward Primer & GCCTCGTCTCATAGACAAGATGGT \\
\hline & Reverse Primer & GAAGGCAGCCCTGGTAACC \\
\hline
\end{tabular}

\section{Western blot analysis}

Uterine tissues were sufficiently ground with liquid nitrogen and then lysed in RIPA lysis buffer (Catalog: P0013B; Bioworld Technology, Inc.) premixed with phenylmethanesulfonyl fluoride. The lysate was centrifuged at $12000 \mathrm{r}$ for $5 \mathrm{~min}$ at $4{ }^{\circ} \mathrm{C}$ to remove the supernatant. Proteins were separated using $12 \%$ sodium dodecyl sulfate-polyacrylamide gel electrophoresis (Catalog: P0014A; Bioworld Technology, Inc.) and then transferred onto polyvinylidene fluoride (PVDF) (Catalog: IPVH00010; Millipore, Co., Ltd.) membranes. The membranes were, then, blocked with $5 \%$ nonfat milk in tris-buffered saline with Tween20 (TBST) for $2 \mathrm{~h}$ and incubated with the primary antibodies overnight at $4{ }^{\circ} \mathrm{C}$. The specific primary antibodies used were as follows: rabbit anti-rat wt-p53 polyclonal antibody (1:1000 dilution; Catalog: bsm-2346M; Bioss, Co., Ltd.), rabbit anti-rat P21 polyclonal antibody (1:500 dilution; Catalog: BS1269; Bioworld Technology, Inc.), rabbit anti-rat cyclin E1 polyclonal antibody (1:1000 dilution; Catalog: BS1085; Bioworld Technology, Inc.), rabbit anti-rat cyclin D1 polyclonal antibody (1:1000 dilution; Catalog: BS2436; Bioworld Technology, Inc., rabbit anti-rat CDK2 polyclonal antibody (1:1000 dilution; Catalog: BS2263; Bioworld Technology, Inc.), rabbit anti-rat CDK4 polyclonal antibody (1:1000 dilution; Catalog: MB0027; Bioworld Technology, Inc.), rabbit anti-rat CDK6 polyclonal antibody (1:1000 dilution; Catalog: 
BS6559; Bioworld Technology, Inc.), rabbit anti-rat pRB polyclonal antibody (1:1000 dilution; Catalog: bs1347R; Bioss, Co., Ltd.), and rabbit anti-rat glyceraldehyde 3-phosphate dehydrogenase (GAPDH) polyclonal antibody (1:5000 dilution; Catalog: ab9485; Abcam, Co., Ltd.). The membranes were washed with TBST and incubated with HRP-conjugated secondary antibodies (goat anti-rabbit IgG [1:10000 dilution; Catalog: ZB-2305; ZSGB-BIO, Co., Ltd.]) for $1 \mathrm{~h}$ at room temperature. An enhanced chemiluminescence substrate kit (Catalog: PE0020; Solarbio, Co., Ltd.) was used to detect HRP activity. After developing and fixing, the film was scanned and analyzed using the Image $\mathrm{J} 2.0$ software to detect the gray values of each protein band.

\section{Statistical analysis}

Statistical analysis was performed using SPSS (version 23.0; IBM Corp.). Normally distributed data are presented as the mean \pm SEM or the mean $\pm S D$, and analyzed using one-way analysis of variance with Fisher's least significant difference (ANOVA-LSD) test or Independent sample $t$ test. $P$ values of $<0.05$ were considered statistically significant. Graphs were generated using PRISM (version 8.0; GraphPad, Inc.).

\section{Results}

\section{Comparison of the abortion rate and morphology of decidual tissues on GD8}

First, we compared the abortion rates of the NP and URSA groups on GD8. In the NP group, the number of normal embryos was 7-9, almost no absorbed and immature embryos or only one was observed. However, in the URSA group, the total number of embryos was 8-10. However, all embryos were immature, and 3-6 embryos were absorbed or dead (Figure 1A). The abortion rateon GD8 in the URSA group was $40.48 \pm 5.43 \%$, which was significantly higher than that in the NP group $(11.07 \pm 2.79 \%)$ (Figure 1B). FollowingH\&E staining, the texture of URSA group tissueswas loose with uneven staining, and large hemorrhagic and necrotic areas were present. Magnified images of the necrotic area (200x and $400 \times$ )showed that the structure of necrotic cells and the boundary between necrotic cells and decidual tissue were unclear(Figure 1C).

Figure 1. A. On GD8, the uterus of the NP group exhibited normal shape with clearly visible implanted embryos. Immature, absorbed, or dead implanted embryos were often found in the URSA group. Arrowheads point to absorbed and necrotic sites. B. The abortion rate (mean $\pm S E M, \%)$ on GD8 was significantly higher in the URSA group compared to that in the NP group. Abortion rate = ratio of absorbed and necrotic embryos to the total number of implanted embryos. *Significantly different $(p<0.005)$ between null vs. NP.; C. 50x, 200x, and 400x magnification images of enucleated decidual tissue stained for hematoxylin and eosin taken after whole tissue scanning. Arrowheads point to necrotic sites. GD8, gestation day 8; NP, normal pregnancy; URSA, unexplained recurrent spontaneous abortion. 


\section{Temporal changes of PRL protein expression in decidual tissues from GD4 to GD8}

To better observe the temporal changes of PRL protein expression during decidualization in URSA mice, we examined the spatiotemporal expression of PRL protein from GD4 (the start time of decidualization) to GD8 (the end time of decidualization) in both groups of tissues. A heightened PRL expression was noted in NP tissues on GD6-GD8, in contrast, a moderate expression, lower than that in NP tissues was observed in URSA tissues. Furthermore, this pattern of expression was enhanced with the progression of pregnancy.

The results of ELISA showed that the expression level of PRL in the NP and URSA groups was similar on GD4 and GD5, an early time of decidualization. However, unlike the continuous increase of PRL expression in the NP group, PRL expression in the URSA group decreased slightly after GD6 and until GD8, the middle and late stages of decidualization. Furthermore, the PRL levels of the two groups were highest on GD6 and a significant difference was apparent from GD6 to GD8. Finally, the URSA decidual tissue exhibited decidualization deficiency.

Figure 2. A. Immunostaining for PRL on GD4-GD8. Brown staining indicates positive signals. Nuclei are counterstained with dark brown. The magnifications in the upper panels are at 200x. Results are shown as the mean $\pm S D$. * Significantly different $(p<0.05)$. B. The histogram on the left shows the difference in PRL levels in decidual tissues between the two groups on GD4-GD8. * Significantly different $(p<0.05)$. The line chart on the right shows the temporal changes of PRL levels. PRL, prolactin; GD, gestation day.

\section{Cyclin-CDK expression during decidualization}

To determine the cause of deficient decidualization in URSA mice, we measured the levels of cell cycle regulatory genes in decidual tissues throughout the whole decidualization process. The results revealed that these genes played an important role in decidualization. Firstly, we found that the gene levels of the NP and URSA groups showed different trends mainly after GD5. The cyclin D levels of the NP group tended to be stable after reaching their peak on GD6, whereas those in the URSA group gradually decreased after GD5. The CDK6 levels of the NP group gradually increased from GD4 to GD8, whereas those of the URSA group were relatively stable and did not significantly increase. Although the levels of cyclin E, CDK4 and CDK2 mRNA in the two groups gradually decreased after reaching their peak on GD5, the degree of decline in the URSA group was significantly greater than that in the NP group (Figurer 3A). Further, by comparing the time associated with significant differences in these gene levels, we found that except for the significant difference between the two groups in the cyclin E mRNA levels beginning on GD7, significant differences in the mRNA expression of other genes began on GD6 (Figure 3B). In general, the gene level differences between the URSA and NP groups occurred in the middle and late stages of decidualization. Consistent with the above results, further quantitative analyses of cell cycle regulatory genes (cyclin D, cyclin E, CDK2, CDK4, CDK6) using western blotting also revealed significant differences 
between the two groups in the levels of all target proteins on GD7-GD8, whereas only cyclin D, CDK4 and CDK6 levels showed significant differences on GD6 (Figure 4).

\section{Discussion}

Endometrial decidualization refers to the process during which ESCs are gradually transformed into DSCs following stimulation by decidualization-inducing factors, and continue to proliferate and spread until the decidua is completely formed [6-9]. Unlike humans, who can spontaneously undergo decidualization without pregnancy, the decidualization process of rats can occur only after embryo implantation $[13,14]$. Generally, at 22-24 days of gestation, after the blastocyst reaches the luminal epithelium and adheres to the implantation site, ESCs start to activate the decidualization process under the action of hormones [15, 16]. ESCs surrounding the blastocyst, including fibroblasts, first begin to differentiate and proliferate until the formation of the primary decidual zone (PDZ), composed of dense DSCs on GD6. Subsequently, DSCs in the PDZ region and adjacent ESCs continue to proliferate and differentiate, and finally form a secondary decidual zone (SDZ) wrapping the PDZ on GD8 [17]. At this point, the 4-day decidualization process is completed, and the mouse endometrium is completely transformed into the pregnant decidua. Whether the decidualization process is smooth or not is closely related to the pregnancy outcome. Decidualization defects will cause a variety of adverse pregnancy outcomes, such as spontaneous abortion, premature delivery, and recurrent abortion [10]. In this study, we selected to use an internationally recognized URSA animal model (CBA / J female * DBA / 2 male) [18]. It was found that the level of decidualization markers in the decidua of URSA mice was significantly lower than that of normal decidua [4], which confirmed that decidualization defects were closely related to the occurrence of URSA disease. Furthermore, this is consistent with the research results of Blois $s \mathrm{~m}$ and achache $\mathrm{h}[11,12]$.

The decidualization process is actually the transformation and proliferation of endometrial stromal cells, which needs to be regulated by a large number of cell cycle regulators. The cell cycle is divided into the interphase (g phase) and division phase (M phase). Most of the cell life activities occur in the interphase, and the division phase accounts for only a small part of cell activities [221]. Although the interphase includes the $\mathrm{G} 1$ phase (first gap), the S phase (synthesis) and G2 phase (second gap), the duration of the G1 phase is long, and the cell cycle arrest in G1 is reversible. When stimulated by certain stimuli, the cell cycle process can be restored again and the cell enters the S phase [222-224]. Cyclin D, cyclin E, CDK4, CDK6 and CDK2 are key cytokines that play a regulatory role in the $\mathrm{G} 1$ phase $[225,226]$. Therefore, this study deeply explored the dynamic changes and roles of the above cytokines in the decidualization process of URSA using animal experiments. According to previous studies, cyclin D accumulates in the G1 phase and forms cyclin D/CDK complexes after binding to CDK4 or CDK6, which can accelerate the initiation of DNA replication and play an important role in promoting cell cycle proliferation. The synthesis of cyclin E in $\mathrm{G} 1$ occurs slightly later than that of cyclin $\mathrm{D}$, and its combination with CDK2 can also promote DNA replication and accelerate the completion of the G1-S phase transition [227-230].

Previous experimental studies and literature reports also showed that cyclin D, cyclin E and their specifically-bound CDK complexes changed dynamically during endometrial decidualization, thus 
regulating decidualization involving cell cycle events, such as cell proliferation, differentiation, and apoptosis. According to the research of Zhang Tan [231] and Tan j [232], cyclin E and CDK2 were expressed at low levels in pregnant mice on GD1-GD2, but the expression in the intima gradually increased with the process of decidualization from GD3, and began to decline after reaching its peak on GD5-GD8. In this study, to more accurately display the changes and trends of detection indices, we detected the mRNA and protein levels of cyclin D, CDK4, CDK6, cyclin E and CDK2 in decidual tissue during the whole process of decidualization (from GD4 to GD8). The results showed that during the decidualization of normal female rats, the expression of cyclin E mRNA increased in the decidua from GD4 to GD5, and began to decrease after its peak on GD5 to GD8. The expression trend of CDK2 is almost consistent with that of cyclin $\mathrm{E}$, which is also consistent with the research results of Jian Tan et al. [236], showing that the expression of cyclin E and CDK2 is higher in the decidua around blastocyst embedding and early after implantation, and then decreases. The expression of cyclin D was low on GD4, but then increased rapidly until it reached its peak on GD8. From the overall trend, cyclin D levels continuously increased in the process of decidualization, with a large increase on GD4-GD6 and a small increase on GD7-GD8. The level of CDK4 increased briefly on GD4-GD5, but then gradually decreased. On the contrary, the expression of CDK6 was lower on GD4-GD5, and increased from GD6-GD8. We believe that normal mice accumulate a certain amount of cyclin E and CDK2 in the endometrium before implantation on GD4. Cyclin E/CDK2 can accelerate the entry of cells into the S phase, promote the proliferation of ESC and increase endometrial receptivity under the synergistic action of other factors, which is conducive to embryo implantation. After the decidualization reaction induced by embryo implantation, the levels of cyclin E and CDK2 increased on GD5, while those of cyclin D and CDK4 also increased on GD4-GD5. The two cyclin/CDK complexes worked together to accelerate the G1-S phase transition of DSC cells around the embryo implantation site and accelerated the proliferation of DSC cells, so the PDZ was quickly formed and blastocysts were embedded. However, after GD6, with the formation of PDZ and the formation of SDZ on GD7 and GD8, cyclin D levels in the PDZ almost disappeared, which inhibited the activity of the cyclin/CDK complex, and affected cell cycle transition from the $\mathrm{G} 1$ to the $S$ phase. However, because the level of cyclin D in the SDZ is still high, the cyclin D levels in the decidua still rise slightly, and the gradual rise of CDK6 levels compensates for the decline of CDK4 levels, maintaining the activity of the cyclin D/CDK complex. Therefore, although some decidual cells continue to proliferate, some cells return to the G1-S phase. These cells break away from the cell proliferation cycle and continue to replicate in the nucleus, maintaining gene stability, limiting the life span of decidual cells, and mediating the orderly apoptosis of decidual cells, thus providing space for embryonic growth. In conclusion, we found that the cyclin/CDK levels showed an overall upward trend in the decidualization process of normal pregnant female rats, which was conducive to maintaining pregnancy.

After clarifying the change trend of cytokines in the decidua of NP mice, we detected and compared the above factors in the decidua of URSA pregnant mice using RT-PCR and Western blot. Firstly, we found that the levels of the above factors decreased in varying degrees compared to those of the normal decidua in the decidualization process of pregnant female rats with URSA. Compared to the level of NP rats, the level of cyclin E in URSA rats showed a more significant increase after GD5. However, according 
to the results of RT-PCR, the mRNA level of cyclin E in URSA female rats and normal female rats was significantly different on GD7 and GD8 $(P<0.05)$. According to the Western blot results, the protein level of cyclin $E$ is consistent with the trend shown using PCR. The protein level of CDK2 was significantly different from that of NP rats on GD7 and GD8 $(P<0.05)$. This significant difference occurred later than GD6 based on the PCR results. We believe that this may be due to a low protein detection sensitivity associated with the Western blot procedure or a delay of mRNA transcription. However, in terms of the overall change trend, the expression of cyclin E and CDK2 in the decidua of URSA pregnant rats was slightly lower than that of NP rats, which had a certain impact on early cell proliferation, differentiation and PDZ formation, but this impact was small and did not affect the early implantation process of embryos. With the advancement of decidualization, the levels of cyclin E and CDK2 continued to decline, so the transformation from the $\mathrm{G} 1$ to the $\mathrm{S}$ phase could not be promoted during the proliferation of SDZ after GD6, affecting cell proliferation. In addition, the levels of cyclin D and CDK4 showed a downward trend after the formation of the PDZ on GD6-GD8, while CDK6 levels did not rise on GD6-GD8 like in normal female rats, but showed a continuous low expression throughout the decidualization process. This low level could not compensate for the maintenance of the activity of the cyclin D/CDK complex, so the cell cycle process was blocked, cells could not transition from the $\mathrm{G} 1$ to the S phase, and stagnated in the G1 phase for a long time, resulting in cell cycle arrest. The formation of PDZ and SDZ was affected, which eventually affected the process of decidualization and caused decidualization defects.

\section{Conclusions}

In conclusion, this study demonstrates that decidualization defects are an important cause of URSA, and that the abnormal decrease of cell cycle regulators (cyclin D, cyclin E, CDK2, CDK4 and CDK6) in the decidua in the middle and later stages of decidualization is closely related to the decidualization defect.

\section{Availability of data and materials}

The current study was based on the results of relevant published studies.

\section{Abbreviations}

URSA

Unexplained Recurrent Spontaneous Abortion

CDK

Cyclin D-Cyclin-Dependent Kinase

NP

Normal Pregnant

GD4

Gestation Day 4, the Start Time of Decidualization

GD8

Gestation Day 8, the End Time of Decidualization 
RSA

Recurrent Spontaneous Abortion

ESCs

Endometrial Stromal Cells

DSCs

Decidual Stromal Cells

PRL

Prolactin

Day 23

the Mid-Secretory Phase of Each Menstrual Cycle

CDKs

Cyclin D-Cyclin-Dependent Kinases

$\mathrm{Rb}$

Retinoblastoma Protein

GD1

Gestation Day 1

H\&E

Hematoxylin and Eosin

RT-PCR

Reverse Transcription PCR

PVDF

Polyvinylidene Fluoride

TBST

Tween-20

GAPDH

Glyceraldehyde-3-Phosphate Dehydrogenase

PDZ

Primary Decidual Zone

SDZ

Secondary Decidual Zone

G Phase

Interphase

M Phase

Division Phase

G1 Phase

First Gap

S Phase

Synthesis

G2 Phase

Second Gap 


\section{Declarations}

Acknowledgements

We gratefully acknowledge all authors for their contribution.

\section{Funding}

This work was supported by the National Natural Science Foundation of China. [grant number:81574014];

This work was also supported by the Scientific research foundation of Heilongjiang University of traditional Chinese Medicine. [grant number:2019MS34]

\section{Corresponding author}

Correspondence to Xiao-ling Feng.

\section{Ethics declarations}

Ethics approval and consent to participate.

All procedures were approved by the Ethic Committee of Heilongjiang University of Chinese Medicine. (Approval No.: HZYLLBA 201906).

Consent for publication.

Not applicable.

Availability of supporting data.

\section{Competing interests}

The authors have no conflict of interest related to the preparation and submission of this study.

\section{References}

1. M. Zhang, J. Xu, X. Bao, W. Niu, L. Wang, L. Du, N. Zhang, Y. Sun, Association between genetic polymorphisms in interleukin genes and recurrent pregnancy loss - a systematic review and metaanalysis, PLoS ONE.12 (1) (2017) e0169891. doi: 10.1371/journal.pone.0169891.[PMC free article] [PubMed]

2. ESHRE Guideline Group on RPL, Bender Atik R, Christiansen OB, Elson J, Kolte AM, Lewis S, Middeldorp S, Nelen W, Peramo B, Quenby S, Vermeulen N, Goddijn M,ESHRE guideline: recurrent pregnancy loss, Hum Reprod Open 2018.2018(2):hoy004. doi:10.1093/hropen/hoy004. [PMC free article] [PubMed] 
3. Wilczynski JR, Radwan P, Tchorzewski H, Banasik M, Immunotherapy of patients with recurrent spontaneous miscarriage and idiopathic infertility: does the immunization-dependent Th2 cytokine overbalance really matter? Arch Immunol Ther Exp. 2012;60(2):151-160. doi:10.1007/s00005-0120161-6. [PubMed] [Google Scholar]

4. Kwak-Kim J, Park JC, Ahn HK, Kim JW, Gilman-Sachs A, Immunological modes of pregnancy loss,Am J Reprod Immunol.2010;63(6):611-623. doi:10.1111/j.1600-0897.2010.00847.x. [PubMed] [Google Scholar]

5. Santamaria X,Taylor $H$, MicroRNA and gynecological reproductive diseases, Fertility and Sterility. 2014;101(6):1545-1551. doi: 10.1016/j.fertnstert.2014.04.044. [PubMed] [CrossRef] [Google Scholar]

6. Lee CH, Kim TH, Lee JH, Oh SJ, Yoo JY, Kwon HS, Kim YI, Ferguson SD, Ahn JY, Ku BJ, Fazleabas AT, Lim JM, Jeong JW, Extracellular Signal-Regulated Kinase 1/2 Signaling Pathway Is Required for Endometrial Decidualization in Mice and Human, PLoS One.2013; 8(9): e75282. doi: 10.1371/journal.pone.0075282.[PMC free article] [PubMed]

7. Asgerally T Fazleabas, Zuzana Strakova,Endometrial function: cell specific changes in the uterine environment,Mol Cell Endocrinol.2002 Jan 25;186(2):143-7. doi:10.1016/S0303-7207(01)00655-4. [PubMed] [Google Scholar]

8. Lee KY, DeMayo FJ,Animal models of implantation,Reproduction.2004 Dec;128(6):679-95. doi:10.1530/rep.1.00340. [PubMed] [Google Scholar]

9. Robbert P Berkhout, Cornelis B Lambalk, Sjoerd Repping, Geert Hamer,Sebastiaan Mastenbroek,Premature expression of the decidualization marker prolactin is associated with repeated implantation failure, Gynecological Endocrinology.2020 Apr;36(4):360-364. doi:10.1080/09513590.2019.1650344.[PubMed]

10. Okada H, Tsuzuki T, Murata H,Decidualization of the human endometrium,Reprod. Med. Biol,2018;17:220-227. doi: 10.1002/rmb2.12088. [PMC free article] [PubMed] [CrossRef] [Google Scholar]

11. Nicole Lustgarten Guahmich, Gregory Farber, Shiva Shafiei, Dylan McNally, David Redmond, Eleni Kallinos, Heidi Stuhlmann, Daniel Dufort, Daylon James, Carl P Blobel ,Endothelial deletion of ADAM10, a key regulator of Notch signaling, causes impaired decidualization and reduced fertility in female mice, Angiogenesis.2020 Aug;23(3):443-458. doi:10.1007/s10456-020-09723-z.[PubMed]

12. Gellersen B, Brosens IA, Brosens JJ, Decidualization of the human endometrium: mechanisms, functions, and clinical perspectives,Semin Reprod Med.2007 Nov;25(6):445-53. doi:10.1055/s-2007991042. [PubMed] [Google Scholar]

13. Brosens JJ, Parker MG, Mclndoe A, Pijnenborg R, Brosens IA, A role for menstruation in preconditioning the uterus for successful pregnancy,Am J Obstet Gynecol,2009 Jun;200(6):615.e16.doi: 10.1016/j.ajog.2008.11.037. [PubMed] [Google Scholar]

14. Klemmt PA, Carver JG, Kennedy SH, Koninckx PR, Mardon HJ,Stromal cells from endometriotic lesions and endometrium from women with endometriosis have reduced decidualization capacity. 
Fertil Steril. 2006 Mar; 85(3): 564-572. doi:10.1016/j.fertnstert.2005.08.046. [PMC free article] [PubMed] [Google Scholar]

15. Laird SM, Tuckerman EM, Li TC, Cytokine expression in the endometrium of women with implantation failure and recurrent miscarriage. Reprod Biomed Online .2006 Jul;13(1):13-23. doi:10.1016/S1472-6483(11)60582-8. [PubMed] [Google Scholar]

16. Natalia Karpovich,Petra Klemmt, Jung Hye Hwang, J Enda McVeigh, John K Heath, David H Barlow, Helen J Mardon, The production of interleukin-11 and decidualization are compromised in endometrial stromal cells derived from patients with infertility. J Clin Endocrinol Metab.2005 Mar;90(3):1607-12.doi: 10.1210/jc.2004-0868. [PMC free article] [PubMed] [Google Scholar]

17. Ryan IP, Taylor RN (1997) Endometriosis and infertility: new concepts. Obstet Gynecol Surv.1997 Jun;52(6):365-71. doi:10.1097/00006254-199706000-00021. PubMed: 9178310. [PubMed] [Google Scholar]

18. Cha J, Sun X. \& Dey S. K, Mechanisms of implantation: strategies for successful pregnancy,Nat Med.2012;18, 1754-1767. doi: 10.1038/nm.3012.[PMC free article] [PubMed] [Google Scholar]

19. Okada H, Tsuzuki T, Murata H. Decidualization of the human endometrium. Reprod. Med. Biol. 2018;17:220-227. doi: 10.1002/rmb2.12088. [PMC free article] [PubMed] [CrossRef] [Google Scholar]

20. Aiwen Le,Zhong Wang,Xiao Dai,Tian Xiao,Rong Zhuo,Baozhen Zhang,Zhonglin Xiao,Xiujun Fan,Icaritin inhibits decidualization of endometrial stromal cells, Exp Ther Med.2017 Dec;14(6):5949-5955. doi: 10.3892/etm.2017.5278. [PubMed][PMC]

21. Mayumi Mori,Agnes Bogdan,Timea Balassa, Timea Csabai,Júlia Szekeres-Bartho,The decidua-the maternal bed embracing the embryo-maintains the pregnancy,Semin Immunopathol. 2016; 38(6): 635-649.doi: 10.1007/s00281-016-0574-0.[PubMed][PMC]

22. Das S. K,Regional Development of Uterine Decidualization: Molecular Signaling by Hoxa10,Molecular Reproduction and Development.2010 May;77(5):387-96. doi: 10.1002/mrd.21133. [PMC free article] [PubMed] [Google Scholar]

23. Kevin $Y$ Lee, Jae-Wook Jeong, Jinrong Wang, Lijiang Ma, James F Martin, Sophia $Y$ Tsai, John $P$ Lydon, Francesco J DeMayo,Bmp2 is critical for the murine uterine decidual response. Molecular and cellular biology.2007 Aug;27(15):5468-78. doi: 10.1128/MCB.00342-07.[PMC free article] [PubMed] [Google Scholar]

24. Roberts JM ,Evolving ideas about cyclins,Cell.1999 Jul 23;98(2):129-32. doi: 10.1016/s00928674(00)81007-7. [PubMed]

25. Sanjoy K Das,Cell cycle regulatory control for uterine stromal cell decidualization in implantation,Reproduction.2009 Jun;137(6):889-99. doi: 10.1530/REP-08-0539.[PubMed]

26. Geum D, Sun W, Paik SK, Lee CC \& Kim K,Estrogen-induced cyclin D1 and D3 gene expressions during mouse uterine cell proliferation in vivo: differential induction mechanism of cyclin D1 and D3,Molecular Reproduction and Development.1997 Apr;46(4):450-8. doi:10.1002/(SICl)10982795(199704)46:4<450::AID-MRD2>3.0.C0;2-N. 
27. Resnitzky DM, Gossen H, Bujard H \& Reed SI,Acceleration of the G1/S phase transition by expression of cyclins D1 and E with an inducible system,Molecular and Cellular Biology.1994 Mar;14(3):166979. doi: $10.1128 / \mathrm{mcb} .14 .3 .1669 .[P M C$ free article] [PubMed]

\section{Figures}

\section{Figure 1}

A. On GD8, the uterus of the NP group exhibited normal shape with clearly visible implanted embryos. Immature, absorbed, or dead implanted embryos were often found in the URSA group. Arrowheads point to absorbed and necrotic sites. $B$. The abortion rate (mean \pm SEM, \%) on GD8 was significantly higher in the URSA group compared to that in the NP group. Abortion rate = ratio of absorbed and necrotic embryos to the total number of implanted embryos. *Significantly different $(p<0.005)$ between null vs. NP.; C. $50 x$, 200x, and 400x magnification images of enucleated decidual tissue stained for hematoxylin and eosin taken after whole tissue scanning. Arrowheads point to necrotic sites. GD8, gestation day 8; NP, normal pregnancy; URSA, unexplained recurrent spontaneous abortion.

\section{Figure 2}

A. Immunostaining for PRL on GD4-GD8. Brown staining indicates positive signals. Nuclei are counterstained with dark brown. The magnifications in the upper panels are at 200x. Results are shown as the mean $\pm S D$. * Significantly different $(p<0.05)$. B. The histogram on the left shows the difference in PRL levels in decidual tissues between the two groups on GD4-GD8. * Significantly different $(p<0.05)$. The line chart on the right shows the temporal changes of PRL levels. PRL, prolactin; GD, gestation day.

\section{Figure 3}

Quantitative RT-PCR analyses of the cell cycle regulatory genes cyclin D, cyclin E, CDK2, CDK4, CDK6 and of the control gene GAPDH from GD4-GD8 in the NP and URSA groups. A. The line charts on the left show the temporal changes of gene expression from GD4 to GD8. B. The bar graphs on the right show the mRNA expression difference in decidual tissues between the two groups on GD4-GD8. * Significantly different ( $p<0.05)$. RT-PCR, reverse transcription PCR; GAPDH, glyceraldehyde 3-phosphate dehydrogenase; CDK, cyclin-dependent kinase; GD, gestation day; NP, normal pregnancy; URSA, unexplained recurrent spontaneous abortion. 


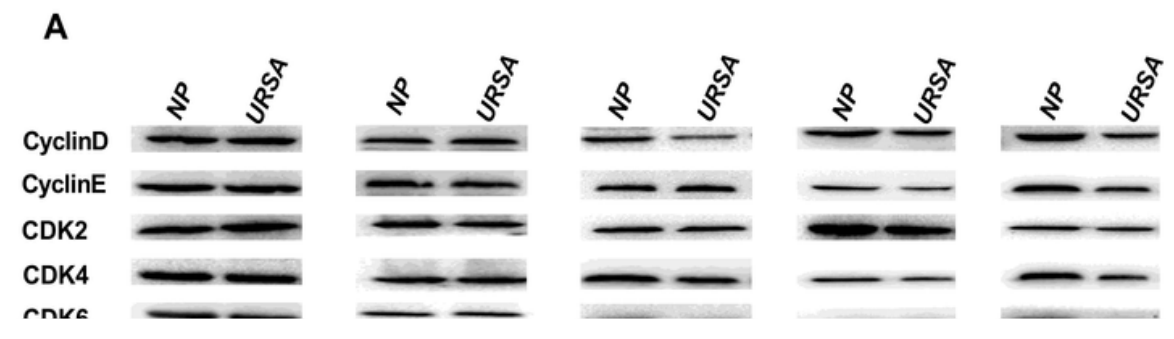

\section{Figure 4}

A. Western blot analyses of cyclin D, cyclin E, CDK2, CDK4, CDK6 and GAPDH in decidual tissues from GD4 to GD8 between the NP and URSA groups. B. Bar graphs showing the quantitative analysis of the protein expression levels of these cytokines based on the Western blot analyses. Results are shown as the mean $\pm \mathrm{SD}$. * Significantly different $(\mathrm{p}<0.05)$. GAPDH, glyceraldehyde 3-phosphate dehydrogenase; CDK, cyclin-dependent kinase; GD, gestation day; NP, normal pregnancy; URSA, unexplained recurrent spontaneous abortion. 